Review Article

\title{
Advances in Research on the circRNA-miRNA-mRNA Network in Coronary Heart Disease Treated with Traditional Chinese Medicine
}

\author{
Fei Lin ${ }^{(D)},{ }^{1}$ Heng-Wen Chen $\left(\mathbb{D},{ }^{2}\right.$ Guo-An Zhao $\left(\mathbb{D},{ }^{1}\right.$ Yan Li, ${ }^{1}$ Xuan-Hui He $\mathbb{D}^{2},{ }^{2}$ \\ Wan-Qian Liang, ${ }^{1}$ Zhuo-Lin Shi, ${ }^{1}$ Si-Yu Sun, ${ }^{1}$ Pan-Pan Tian $\mathbb{D}^{2},{ }^{2}$ Ming-Yan Huang $\mathbb{D}{ }^{2}$ \\ and Chao Liu $\mathbb{D i D}^{2}$ \\ ${ }^{1}$ Department of Cardiology, The First Affiliated Hospital of Xinxiang Medical University, Xinxiang 453100, Henan, China \\ ${ }^{2}$ Department of Cardiology, Guang'anmen Hospital, China Academy of Chinese Medical Sciences, Beijing 100053, China
}

Correspondence should be addressed to Guo-An Zhao; guoanzhao@xxmu.edu.cn

Received 14 September 2019; Revised 9 December 2019; Accepted 19 December 2019; Published 17 February 2020

Academic Editor: Yoshiki Mukudai

Copyright (C) 2020 Fei Lin et al. This is an open access article distributed under the Creative Commons Attribution License, which permits unrestricted use, distribution, and reproduction in any medium, provided the original work is properly cited.

\begin{abstract}
There has been an increase in morbidity and mortality related to coronary heart disease (CHD) in China in recent years. Numerous clinical experiences and studies have shown that traditional Chinese medicine (TCM) plays an important role in the prevention, treatment, and prognosis of CHD. However, the mechanism of TCM in the treatment of CHD has not yet been elucidated. The circRNA-miRNA-mRNA network consists of miRNA that is competitively bound by circRNA, and miRNA regulates the transcription level of mRNA. Through literature review, we found that the circRNA-miRNA-mRNA network acts to contribute to certain effects to CHD such as myocardial hypertrophy, myocardial fibrosis, and heart failure. TCM contains constituents that act against CHD by antiatherosclerosis and apoptosis inhibition action, cardiac and cardiomyocyte protection, and these components also promote cell growth and protection of the vascular system by regulating miRNAs. Therefore, we consider that the circRNA-miRNAmRNA network may be a new regulatory mechanism for the effective treatment of CHD by TCM.
\end{abstract}

\section{Introduction}

The morbidity and mortality caused by coronary heart disease (CHD) are steadily increasing in China. According to a 2018 report on cardiovascular disease in China, in 2013, $11,396,104$ people died from CHD, with CHD mortality in 2016 of 113.46/100 thousand for urban residents and 118.78/ 100 thousand for rural residents. Therefore, it is urgent to find effective methods for the prevention and treatment of CHD events [1].

The etiology of CHD is cavity vascular stenosis, coronary insufficiency, acute or transient ischemia, and hypoxia of myocardial cells caused by coronary atherosclerosis (AS). $\mathrm{CHD}$ belongs to the XiongBi or heartache category in traditional Chinese medicine (TCM). With a history of thousands of years, TCM is unique in theory and rich in clinical practice. Syndrome differentiation and treatment and holistic concept are two basic characteristics of TCM. A syndrome reflects the pathological essence of a certain stage in the development of disease. Phlegm and blood syndrome (PBSS) is one of the common syndrome types in CHD, and its formation and development are closely related to blood stasis, obesity, hyperlipidemia, hyperglycemia, body deficiency, old age, and environment. For PBSS, a satisfactory clinical outcome is achieved by promoting blood circulation and dispersing phlegm. There are three types of Chinese medicine used for the prevention and treatment of $\mathrm{CHD}$, namely, a single Chinese medicine (e.g., redroot salvia, Rhodiola, and pseudoginseng), effective ingredients (e.g., tanshinone IIA, salvianolic acid, and ligustrazine), and prescriptions (e.g., Xuefu-Zhuyu decoction, Chaihu-Shugan powder, Gualou-Xiebai-Banxia decoction, and Ditan decoction). However, the mechanisms of these Chinese herbal medicines ( $\mathrm{CHMs}$ ) have not been fully elucidated. 
Circular RNAs (circRNA) and micro-RNAs (miRNA) are noncoding RNAs and have been confirmed to contribute to the pathogenesis and progression of $\mathrm{CHD}[2,3]$. CircRNA and miRNA interact with each other and regulate the mRNA expression of critical genes in CHD genesis and progression. Therefore, we speculated that there is a connection between the circRNA-miRNA-mRNA network and TCM and gathered supporting data through reviewing and summarizing the literature.

\section{The Therapeutic Effect of TCM in CHD}

TCM aims to treat CHD based on holistic regulation and syndrome differentiation and treatment. According to the model theory of gene constitution syndrome pattern, the occurrence and development of TCM syndromes are mainly based on the genomics of gene expression, which is a powerful approach to gain insight into the prevention and treatment of diseases [4].

In TCM, CHD is also described as XiongBi, which belongs to the category of chest pain, thoracic obstruction, heartache, or angina pectoris as a consequence of cold invasion, improper diet, mood disorder, fatigue, bodily internal injury, or body deficiency due to old age. CHD entails mainly obstruction of the heart channel with injury to other organs including the lungs, liver, spleen, and kidneys [5]. The syndromes of CHD in Chinese clinical medicine manifest as intermingled deficiency in origin (mostly $Q i$, blood, but also Qi-Yin and Yang) and excess in superficiality (mainly blood stasis and phlegm turbidity, but also cold coagulation and Qi stagnation) [6]. Qi in the TCM is a kind of substance with strong vitality and constant movement and extremely subtle, which is the unity of life substance and physiological function. Yin and Yang represent two opposite aspects of every object and their implicit conflict and interdependence. Generally, anything that is moving, ascending, bright, progressing, and hyperactive, including functional disease of the body, pertains to yang. The characteristics of stillness, descending, darkness, degeneration, and hypoactivity, including organic disease, pertain to Yin.

Traditional Chinese syndromes in $X i o n g B i$ involve (a) heart-blood stasis syndrome (Xin Xue Yu Zu), (b) cold coagulation in heart-vessel syndrome (Han Ning Xin Mai), (c) Qi stagnation blood stasis syndrome (Qi Zhi Xin Xiong), (d) phlegm stasis in heart-vessel syndrome (Tan Zhuo Bi $\mathrm{Zu}$ ), (e) deficiency of both $Q i$ and Yin syndrome (Qi Yin Liang $X u$ ), (f) Yin deficiency of heart and kidney syndrome (Xin Shen Yin Xu), and (g) Yang deficiency of heart and kidney syndrome (Xin Shen Yang $X u$ ). These syndrome types are shown in Table 1 [6].

Based on the principle of holistic regulation and syndrome differentiation and treatment, CHMs have been widely used in disease prevention and treatment in China and have been proven to have great clinical effectiveness on cardiovascular system disease. A previous study that summarized and introduced the application of plant products in $\mathrm{CHD}$, AS, dyslipidemia, and hypertension also sorted formulae and herbs according to specific cardiovascular diseases [7]. The plant products applied in AS and dyslipidemia were Allium fistulosum L. (Amaryllidaceae), Allium sativum L. (Amaryllidaceae), Astragalus propinquus (Fabaceae), Coptis chinensis Franch. (Ranunculaceae), Crataegus spp. (Rosaceae), Epimedium brevicornum Maxim (Berberidaceae), Fallopia multiflora Thunb. (Polygonaceae), Fermentum rubrum (Aspergillaceae), Olea europaea L. (Oleaceae), Panax ginseng C.A. Mey (Araliaceae), Pueraria lobata (Willd) ohwi (Leguminosae), Reynoutria japonica Houtt (Polygonaceae), Rheum palmatum Linn. (Polygonaceae), Salvia miltiorrhiza Bunge (Lamiaceae), Scutellaria baicalensis Georgi (Lamiaceae), Senna obtusifolia Linn. (Fabaceae), Cassia tora Linn. (Leguminosae), Ligusticum chuanxiong Hort. (Umbelliferae), Rhodiola crenulata H. Ohb (Crassulaceae), and Curcuma longa L. (Gingeraceae) [7-10].

Over the last decades, some TCM decoctions have been confirmed to be effective in the treatment of CHD. For instance, combination therapy with Xuefu Zhuyu decoction and traditional antianginal medications (TAMs) was more effective in treating angina pectoris than TAMs alone. Evidence has indicated that Xuefu Zhuyu decoction combined with TAMs was more effective in improving RAS $(\mathrm{RR}=1.29 ; 95 \% \mathrm{CI}=(1.20,1.38))$, ECG $(\mathrm{RR}=1.37$; 95\% CI $=(1.22,1.54))$, and blood HDL-C level $(\mathrm{MD}=0.29 \mathrm{mmol} / \mathrm{L} ; 95 \% \mathrm{CI}=(0.23,0.35))$ compared with TAMs alone from a meta-analysis and systematic review of 14 randomized controlled trials [11]. There was benefit in the use of Tongxinluo (TXL) capsule for secondary prevention after acute myocardial infarction (AMI). A systematic review and meta-analysis of randomized controlled trials indicated that TXL improves cardiac function; regulates blood lipid total cholesterol (TC), triglycerides (TG), and low-density lipoprotein-C (LDL$\mathrm{C}$ ); and decreases the level of hs-C-reactive protein (hsCRP) [12]. A meta-analysis indicated that Huoxue Huayu therapy (HXHY) is an effective and safe therapy for patients after percutaneous coronary intervention (PCI). HXHY had a greater beneficial effect on reducing the instent restenosis (ISR) rate and the degree of restenosis, improving Seattle Angina Questionnaire (SAQ) scores and increasing the revascularization rate compared with placebo. However, the rate of death and myocardial infarction (MI) of patients treated with HXHY was no different from that of those treated with placebo $(P>0.05)$ [13].

According to new studies, the pharmacological mechanisms of CHM are relevant to the effects on vascular smooth muscle cells (VSMCs), endothelial cells, cardiomyocytes, macrophages, and monocytes. Details are shown in Table 2 [7].

\section{Regulation of miRNAs in CHD}

With the improvement in modern living standards, phlegm and blood stasis commonly occur and then aggravate each other during CHD progression $[14,15]$. Phlegm is a pathological substance caused by the disturbance of body fluid. Blood stasis is a form of pathology caused by the disturbance of blood circulation. Research on CHD with PBSS has mainly focused on lipid metabolism, inflammatory factors, hemorheology, coagulation function, endothelial cell injury, 
TABLe 1: Chinese clinical medicine for XiongBi.

\begin{tabular}{lcc}
\hline Syndromes & Therapeutic principle & Formulae \\
\hline & & \\
& \\
Xin Xue Yu & Huo Xue Hua Yu, Tong & Xuefu-Zhuyu decoction \\
$\mathrm{Zu}$ & Mai Zhi Tong
\end{tabular}

$\begin{array}{lc}\text { Qi Zhi Xin } & \text { Shu Gan Li Qi, Huo } \\ \text { Xiong } & \text { XueTong Luo }\end{array}$

Xiong

Tan Zhuo Bi Tong Yang Xie Zuo, $\mathrm{Zu} \quad$ Huo Tan Xuan Bi

Han Ning Xin Xin Wen San Han, Xuan Mai Tong Xin Yang

$\begin{array}{lc}\text { Qi Yin Liang } & \text { Yi Qi Yang Yin, Huo } \\ \mathrm{Xu} & \text { Xue Tong Mai }\end{array}$

Xin Shen Yin Zi Yin Qing Huo, Yang $\mathrm{Xu} \quad \mathrm{Xin} \mathrm{He}$ Luo

Xin Shen

Yang Xu
Chaihu-Shu gan San

(1) Gualou-Xiebai-

(2) Di-Tan decoction
Banxia decoction;

Taoren (Prunus persica (L.) Batsch), Honghua (Carthamus tinctorius L.), Danggui (Angelica sinensis (Oliv.) Diels), ShengDihuang (Rehmannia glutinosa Libosch.), Niuxi (Achyranthes bidentata Bl.), Chuanxiong (Ligusticum chuanxiong Hort.), Jiegeng (Platycodon, grandiflorum (Jacq.) A.DC.), Chishao (Paeonia lactiflora PalL), Zhike (Citrus aurantium L.), Gancao (Glycyrrhiza uralensis Fisch.), Chaihu (Bupleurum chinense)

Chenpi (Citrus reticulata), Chaihu (Bupleurum chinense), Chuanxiong (Ligusticum chuanxiong Hort.), Xiangfu (Cyperus rotundus), Zhike (Citrus aurantium L.), Shaoyao (Paeonia lactiflora Pall), Gancao (Glycyrrhiza uralensis Fisch.)

(1) Gualou (Trichosanthes kirilowii Maxim.), Xiebai (Allium macrostemon Bge.), Jiangbanxia (Rhizome Pinelliae Preparata), Huangju (rice wine);

(2) Fuling (Poria cocos (Schw.) Wolf), Renshen (Panaxginseng C.A.Mey.), Gancao (Glycyrrhiza uralensis Fisch.),Chenpi (Citrus reticulata Blanco), Danxing (Arisaema Cum Bile), JiangBanxia (Rhizome Pinelliae Preparata), Zhuru (Caulis Bambusae in Taenia), Zhishi (Citrus aurantium L.), Shichangpu (Acorus tatarinowii Schott) (1) Zhishi (Citrus aurantium L.), Houpu (Magnolia officinalis Rehd. et Wils.), Xiebai (Allium macrostemon Bge.), Guizhi (Cinnamomum cassia Presl), Gualou (Trichosanthes kirilowii Maxim.);

$$
\text { (2) Danggui }
$$

(1) Zhishi-Xiebai-Guizhi decoction

(2) Si-Ning decoction

(1) Shengmai San, (2) Renshen -Yang-Rongdecoction
1. Tian-Wang-Bu-Xin Dan,

2. Zhi-Gancaodecoction
(Angelica sinensis (Oliv.) Diels), Guizhi (Cinnamomum cassia Presl), Shaoyao(Paeonia lactiflora Pall), Xixin (Asarum heterotropoides), Tongcao

(Tetrapanax papyrifer (Hook.) K. Koch), Gancao (Glycyrrhiza uralensis Fisch.), Dazao (Ziziphus jujuba Mill.)

(1) Renshen (Panax ginseng C.A.Mey.), Maimendong (Ophiopogon japonicus), Wuweizi (Schisandra chinensis (Turcz.) Baill.);

(2) Renshen (Panax ginsengC.A.Mey.), Baizhu (Atractylodes macrocephala Koidz.Atractylodes macrocephala), Fuling (Koidz.Atractylodes macrocephala Koidz.), Gancao (Glycyrrhiza uralensis Fisch.), Chenpi (Citrus reticulata), Huangqi (Astragalus membranaceus (Fisch.) Bge.), Danggui (Angelica sinensis (Oliv.) Diels), Baishao (Paeonia lactiflora Pall.), ShuDihuang (Rehmannia glutinosa), Wuweizi (Schisandra chinensis (Turcz.) Baill.), Guixin (Cinnamomum cassia Presl), Zhiyuan (Polygala tenuifolia Willd.)

(1) Renshen (Panax ginseng C.A.Mey.), Fuling (Poria cocos (Schw.) Wolf), Xuanshen (Scrophularia ningpoensis Hemsl.), Danshen (Salvia miltiorrhiza Bge.), Jiegeng (Platycodon grandiflorum (Jacq.) A.DC.), Yuanzhi (Polygala tenuifolia Willd.), Danggui (Angelica sinensis (Oliv.) Diels), Wuweizi (Schisandra chinensis (Turcz.) Baill.), Maimendong (Ophiopogon japonicus), Tianmendong (Asparagus cochinchinensis), Baiziren (Platycladus orientalis (L.) Franco),

Suanzaoren (Semen Ziziphi Spinosae), ShengDihuang (Rehmannia glutinosa Libosch.);

(2) Gancao (Glycyrrhiza uralensis Fisch.), Shengjiang (Zingiber officinale Rosc.), Guizhi (Cinnamomum cassia Presl), Renshen (Panax ginseng C.A.Mey.), ShengDihuang (Rehmannia glutinosa Libosch.), Ejiao (Asini Corii colla), Maimendong (Ophiopogon japonicus), Maren (Cannabis sativa L.), Dazao (Ziziphus jujuba Mill.)

(1) Renshen (Panax ginseng C.A.Mey.), Fuzi (Aconitum carmichaelii Debx.);

(2) ShuDihuang (Rehmannia glutinosa), Shanyao (Dioscotea opposita Thunb), Shanzhuyu (Cornus officinalis Sieb.et Zucc.), Gouqizi (Lycium barbarum L.), Gancao (Glycyrrhiza uralensis Fisch.), Duzhong (Eucommia ulmoides Oliv.), Rougui (Cinnamomum cassia Presl), ZhiFuzi (Aconitum carmichaelii Debx.) 
TABLE 2: The pharmacological mechanisms of CHM.

\begin{tabular}{|c|c|c|}
\hline VSMCs & Endothelial cells & Cardiomyocytes \\
\hline $\begin{array}{l}\text { Inhibiting expression or activity of contractile and } \\
\text { structural proteins }\end{array}$ & Activation of NO signaling pathway & $\begin{array}{c}\text { Alleviation of cardiac } \\
\text { hypertrophy }\end{array}$ \\
\hline Regulating expression of extracellular matrix proteins & Inhibition of inflammation & Attenuation of oxidative stress \\
\hline $\begin{array}{l}\text { Regulating calcium levels in a PKA/PKG/PKC- } \\
\text { dependent way }\end{array}$ & Attenuation of oxidative stress & Inhibition of apoptosis \\
\hline Attenuating proliferation and migration of VSMCs & Inhibition of apoptosis, & Opening $\mathrm{K}_{\mathrm{ATP}}$ channels \\
\hline $\begin{array}{l}\text { Anti-inflammation improving mitochondrial } \\
\text { function }\end{array}$ & $\begin{array}{l}\text { Inducing angiogenesis suppression of endothelial } \\
\text { permeability }\end{array}$ & ANP secretion \\
\hline
\end{tabular}

endoplasmic reticulum autophagy, insulin resistance, genomics, proteomics, and metabolomics [16].

MiRNAs are nuclear genome-encoded single-stranded small (18-23 bases) RNAs that are highly expressed in heart tissue, and they play central roles in the miRNA-miRNA functional synergistic network [17]. The specificity and timing of miRNA expression are very similar to the dynamic spatiotemporal characteristics of TCM syndromes. The expression of miR-146b-5p, miR-199a-3p, and miR-199a-5p is upregulated, CALR and TP53 are downregulated in blood stasis syndrome, and miR-363-5p and miR-668 are downregulated, and RIPK2 and STK4 are upregulated in phlegm-turbidity syndrome. In PBSS, the expression of miR1207-5p, miR-1321, miR-320d, miR-765, and other miRNAs is upregulated, while the expression of miR-1181, miR-12253p, miR-1248, miR-668, miR-1223, miR-1281, miR-1538, miR-181d, miR-342-3p, miR-483-3p, miR-491-3p, miR-494, miR-532-3p, miR-668, and miR-98 is downregulated [18-20]. Peroxisome proliferator-activated receptor- $\alpha$ (PPAR- $\alpha$ ) is an important factor affecting PBSS that can be regulated by miR-21 [21]. MiR-4433-3p and miR-363-5p are downregulated in patients with $Q i$ deficiency, phlegm, and blood stasis syndrome. In patients with $Q i$ deficiency and blood stasis syndrome, miR-17-5P, miR-320a, and miR-320c are downregulated [22].

The target genes of miRNA related to CHD with PBSS may regulate cell signaling pathways through the transforming growth factor- $\beta$ (TGF- $\beta$ ) signaling pathway. MiRNAs such as miR-107, miR-146b-5p, miR-199a-5p, and miR-661 are upregulated, while miR-1321 is downregulated after HuoxueHuatan Anshen formula intervention in angina pectoris CHD with PBSS [23]. In addition, TGF- $\beta 1$ is the initiating factor for synthesizing and depositing collagen fibers and other extracellular active components. After AMI, miR-21 induces myocardial fibrosis through the TGF-0205/ Smad7 pathway [24], and circRNA-010567 promotes myocardial fibrosis by inhibiting miR-141 via targeting TGF- $\beta 1$ [25]. In addition, abnormal proliferation or migration of VSMCs can lead to vessel lesions, resulting in AS and in stent-restenosis. The expression of miR-378a-5p was increased in the group with stent restenosis compared with healthy people, and miR-378a-5p overexpression promoted proliferation and migration in VSMCs by targeting CDK1 [26]. The changes in VSMC phenotype and vascular calcification are major characteristics of AS. VSMCs express miRNAs, and there is a causal link between miRNAs such
miR-21, miR-125b, miR-133a, miR-136, miR-143/145, miR1 , and miR-206 and the pathogenesis of vascular disorders in cardiovascular disease with diabetes mellitus [27]. It is important that miRNAs such as miRNA-143/-145, miR-133, miR-125a-5p, miR-23b, miR-638, miR-663, miR-21, miR100 , miR-143, and miR-145 can regulate VMSC function, and miR-126-3p, miR-126-5p, miR-92a, miR-221, and miR222 can also regulate endothelial cell function [28, 29].

The various aspects of treatment for CHD with PBSS are also closely related to miRNAs. Treatment of spleen phlegmturbidity and blood stasis syndrome of CHD may be accomplished via multiple targets, including miRNA [30]. Studies have detected 12 differentially expressed miRNAs in dyslipidemia patients with spleen deficiency syndrome, and the results showed 10 downregulated (miR-124-3p, miR-95p, miR-133b, miR-1, miR-136-5p, miR-144-3p, miR-133a, miR-149-5p, miR-200a-3p, and miR-219-5p) and 2 upregulated (miR-193a-5p, miR-665) miRNAs that were involved in fatty acid metabolism, phagocytosis, and proliferation- and apoptosis-related signal pathways [31]. In addition, miRNAs can discriminate the severity of blood stasis syndrome, allowing prognostication for patients with acute coronary syndrome. For example, miR-208a-3p expression can be used to distinguish acute coronary syndrome patients with blood stasis syndrome from healthy volunteers. MiR-222-3p and miR-198 may help to grade the severity of blood stasis [32]. Additionally, it was reported that TCM remedies contain active components that can protect cardiomyocytes, protect against AS, inhibit apoptosis, promote cell growth, and protect the vascular system by regulating miRNAs. Details are shown in Tables 3 and 4.

\section{CircRNA-miRNA-mRNA in CHD}

CircRNA is an endogenous noncoding RNA. It has no $5^{\prime}$ end cap or $3^{\prime}$-end poly (A) tail as a covalently closed loop from precursor mRNA back-splicing by the spliceosome machinery. Some circRNAs have been shown to act as miRNA sponges, to interact with RNA-binding proteins, to regulate transcription, or to be translated into proteins. CircRNAs have been widely explored in antiaging strategies, diabetes, cardiovascular diseases, and malignant tumors [44-50]. In various cardiovascular tissues and organs, circRNA participates in physiological and pathological processes such as myocardial repair regulation and fibrosis of vascular smooth muscle cells and myocardium, without 
TABLE 3: The influence of active ingredients-miRNA in TCM on cardiovascular diseases.

\begin{tabular}{lcccc}
\hline Active components & Cell types & MiRNAs & Targets & Effects (references) \\
\hline & Macrophages & miR-375 & KLF4 & Anti-AS [33] \\
Tanshinone IIA & NRCMs & miR-133 & MAPK ERK1/2 & Protecting cardiomyocytes [34] \\
& H9c2 & miR-152-3p & PTEN & Inhibiting apoptosis [35] \\
Salvianolate & H9c2 & miR-122-5p & Bax, Bcl-2 & Inhibiting apoptosis [36] \\
Astragaloside IV & H9c2 & miR-23a/miR-92a. & PI3K/AKT & Protects rat cardiomyocytes [37] \\
Notoginsenoside R1 & H9c2 & miR-21 & PI3K/AKT & Cardioprotective actions [38] \\
Ginsenoside Rb1 & NRCMs & miR-208 & - & Protecting H/I impaired NRCMs [39] \\
Ginkgolide B & EPC & miR-126 & Akt & Promoted cell growth [40] \\
Crataegus special extract & HUVEC & miR-155 & eNOS & Protecting vascular [41] \\
\hline
\end{tabular}

EPC: endothelial progenitor cells; HUVEC: human umbilical vein endothelial cells; NRCMs: neonatal rat cardiomyocytes.

TABLE 4: The influence of gormulas of TCM-miRNA on cardiovascular diseases.

\begin{tabular}{lccc}
\hline TCM formulas & miRNAs & Targets & Effects (references) \\
\hline Compound Danshen dropping pills & miR-200b & VEGF/TGF- $\beta 1$ & Diabetic cardiomyopathy [42] \\
Fuzheng Huayu Capsule & miR-29 & - & Myocardial fibrosis [43] \\
Xinfukang pill & miR-21 & Fas/FasL & Inhibiting apoptosis [44] \\
Xinfukang pills & miR-1 & Fibullin-2 & Cardiac hypertrophy [45] \\
Qi li qiangxin capsule & niR-199a-5p & - & Cardiac remodeling and hypertrophy [46] \\
\hline
\end{tabular}

being affected by the expression of maternal genes. Thus, circRNAs affect the occurrence of cardiovascular diseases such as heart failure and hypertension. CircRNAs and miRNAs interact with each other in CHD genesis and progression. The circRNA-miRNA network may regulate mRNA participating in the development of CHD through competitive endogenous RNA (ceRNA). A new regulation model of gene expression and a complex network system has been developed that involves ceRNA, in which coding RNA and noncoding RNA interact with each other. This regulatory network is constructed to affect the mutual regulation, restriction, and interaction among lncRNA, circRNA, miRNA, and mRNA, thus affecting the target genes participating in CHD [51].

The circRNAs that have been reported to be differentially expressed in $Q i$ stagnation and blood stasis syndrome include circRNA 9849 , circRNA 1 1523, $\quad \operatorname{circRNA} A_{1} 8046$, and $\operatorname{circRNA} \mathrm{A}_{2} 4450$, with circRNA 11523 being related to hsa-circ-0005860 in circBase [52]. Through the KEGG pathway enrichment analysis of differential circRNA in $Q i$ stagnation and blood stasis syndrome, five enrichment pathways were found, namely, path:hsa05144 (malaria), path:hsa04914 (progesterone-mediated oocyte maturation), path:hsa04650 (natural killer cell-mediated cytotoxicity), path:hsa04110 (cell cycle), and path:hsa05203 (viral carcinogenesis). These circRNAs also participate in the regulation of natural killer cell-mediated cytotoxicity and cell cycle. Previous studies showed that a total of 1670 circRNAs and 13 miRNAs were differentially expressed in AMI, and 110 circRNAs and 11 miRNAs were differentially expressed in CHD. Gene ontology (GO) and pathway analyses for differentially expressed circRNAs showed that many pathways, diseases, and functions participate in the development of AMI [53]. One study also showed that there were nine circRNAs, namely, hsa_circ_0089378, hsa_circ_0083357, hsa_circ_0082824, hsa_circ_0068942, hsa_circ_0057576, hsa_circ_0054537, hsa_circ_0051172, hsa_circ_0032970, and hsa_circ_0006323, that could negatively regulate miR$130 \mathrm{~A}-3 \mathrm{p}$, resulting in the upregulation of transient receptor potential cation channel subfamily M member 3 (TRPM3) in patients with $\mathrm{CHD}$, with nine circRNAs promoting TRPM3 expression by inhibiting hsa-miR-130a-3p in CHD patients [54]. The effects of the circRNA-microNA-mRNA network on CHD include myocardial hypertrophy, myocardial fibrosis, and heart failure, which are shown in Table 5.

Adrb1: adrenoceptor beta 1; Adcy6, adenylate cyclase 6; ARC: activity-regulated cytoskeletal protein; CDR1as: Cerebellar degeneration-related protein 1 antisense ciRS-7; CDIP1: cell death-inducing protein 1; circANRIL: Circular antisense non-coding RNA in the INK4 locus; COL1A1: type I collagen; COL3A1: type III collagen; EGR1: early growth response protein 1; CTGF: connective tissue growth factor; FOXO1: Forkhead Box Protein O1; Gsk3 $\beta$ : glycogen synthase kinase $3 \beta$; IER2: immediate early response protein 2 ; MFACR: mitochondrial fission and apoptosis-related circRNA; MTP18: mitochondrial protein, $18 \mathrm{kDa}$; NCX1 sodium/calcium exchanger 1 (ncxl) gene; NR4A1: nuclear receptor subfamily 4 group A member 1; NRG-1: Neuregulin-1; PARP: Poly (ADP-ribose) polymerase; PES1: pescadillo homologue 1; $\alpha$-SMA: $\alpha$-smooth muscle actin; SP1: transcription factor Sp1; SRF: serum response factor; STIM1: stromal interaction molecule 1; TGF- $\beta 1$ : transforming growth factor- $\beta 1$.

CircRNAs with a covalently closed continuous loop are an abundant class of endogenous RNAs that are formed during the maturation of precursor mRNA, and they have been widely researched with respect to cardiovascular diseases. When CircANRIL binds to pescadillo homologue 1 (PES1), it impairs exonuclease-mediated pre-rRNA processing and ribosome biogenesis in vascular smooth muscle cells and macrophages and induces nucleolar stress and p53 activation, resulting in the induction of apoptosis and inhibition of proliferation for conferring atheroprotection [55]. Cdrlas also functioned as a powerful miR-7a sponge in 
TABLE 5: The influence of PES1 (P) on cardiovascular diseases.

\begin{tabular}{lccc}
\hline circRNA & miRNA (P/N) & MRNA (P/N) & Effect \\
\hline circANRIL & - & PES1 (P) & AS [55] \\
CDR1as & miR-7a (P) & PARP, SP1 (P) & Myocardial infarction [56] \\
circRNA-HRCR & miR-223 (N) & ARC (P) & Myocardial hypertrophy [57] \\
MFACR & miR-625-3p (N) & Apoptosis [58] & Myocardial fibrosis [25] \\
circRNA_010567 & miR-141 (N) & TGF- $\beta 1(\mathrm{P})$ & Apoptosis [59] \\
circNCX1 & miR-133a-3p (N) & CDIP1 (P) & Cell proliferation and differentiation [60] \\
Circ-SATB2 & miR-939 (N) & STIM1 (P) & Migration of vascular smooth muscle [61] \\
circCHFR & miR-370 (N) & FOXO1/Cyclin D1 (N) & Myocardial hypertrophy [62] \\
circSlc8a1 & miR-133a (N) & SRF, CTGF, Adrb1, Adcy6 (P) & Cardiac regeneration [63] \\
circNfix & miR-214 (N) & Gsk3 $(\mathrm{P})$ & Cardiac fibroblasts [64] \\
circHIPK3 & miR-29b-3p (N) & a-SMA, COL1A1, COL3A1 (N) &
\end{tabular}

$\mathrm{P} / \mathrm{N}$ : positive regulation or negative regulation.

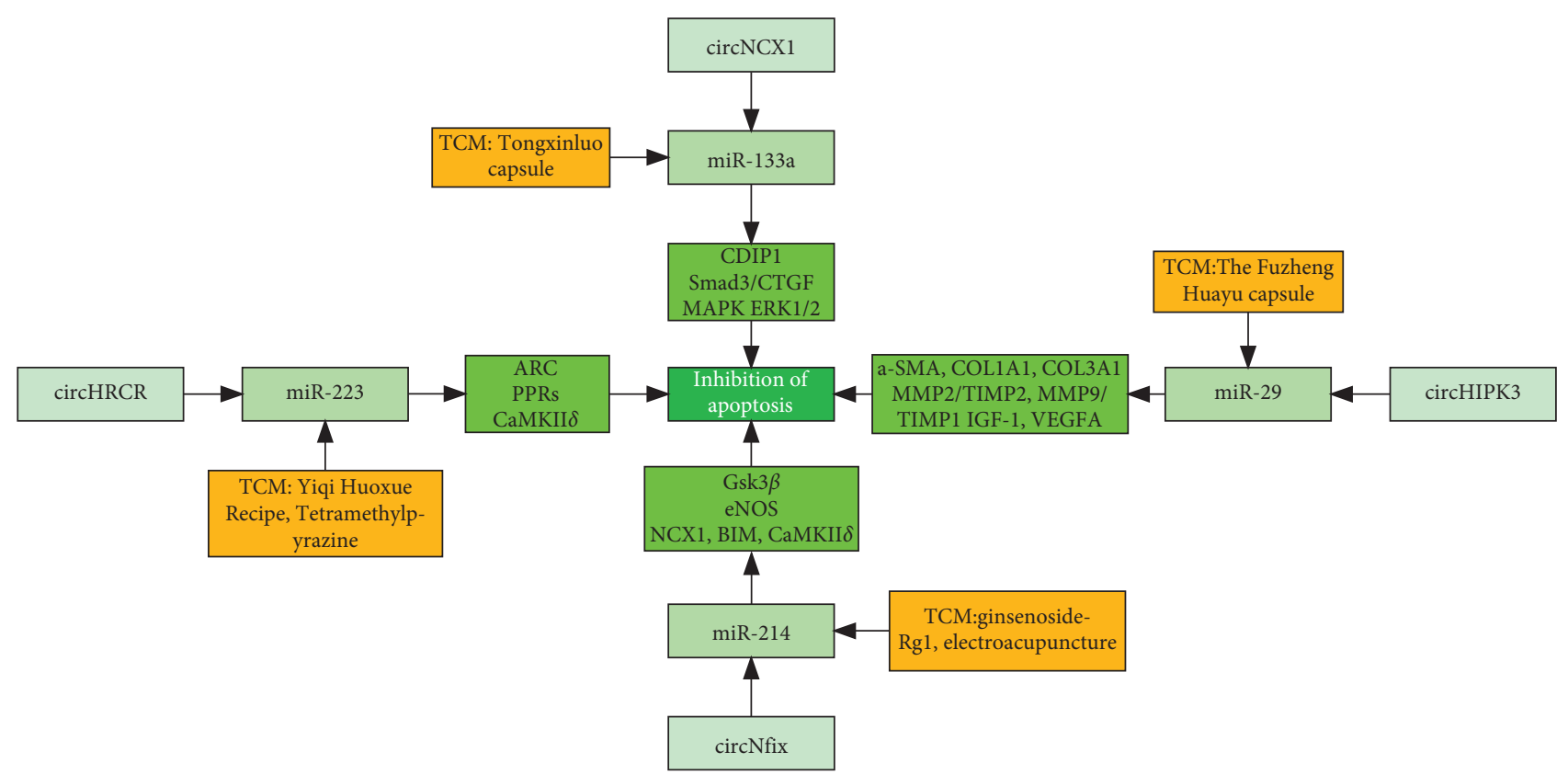

FIgURE 1: The potential role of the circRNA-miRNA-mRNA regulatory network in the cardiovascular diseases treated with TCM CDIP1: cell death-inducing protein 1; Smad3: recombinant human mothers against decapentaplegic homolog 3; CTGF: connective tissue growth factor; MAPK: mitogen-activated protein kinase; ERK1/2: extracellular signal regulated kinases 1 and 2; ARC: recombinant activity regulated cytoskeleton associated protein; PPRs: pentatricopeptide repeat proteins; CaMKII $\delta$ : calmodulin-dependent protein kinase II $\delta$; GSK3 $\beta$ : glycogen synthase kinase-3 $\beta$; eNOS: endothelial nitric oxide synthase; NCX1: sodium/calcium exchanger 1; BIM: BCL2-like 11; aSMA: anti- $\alpha$ smooth muscle actin; COL1A1: recombinant collagen type I alpha 1; COL3A1: recombinant collagen type III alpha 1; MMP: mitochondrial membrane potential; TIMP: tissue inhibitor of metalloproteinase; IGF-1: insulin-like growth factor-1; and VEGFA: vascular endothelial cells growth factor A.

myocardial cells, involving the function of miR-7a targets PARP and SP1 in MI injury [56]. CircRNA HRCR can sequester and inhibit miR-223 activity and was then used in the treatment of cardiac hypertrophy and heart failure through increasing the ARC expression [57]. MFACR regulates mitochondrial dynamics and apoptosis through miR-652-3p and MTP18 [58]. CircRNA_010567 promotes myocardial fibrosis via suppressing miR-141 by targeting TGF- $\beta 1$ [25]. CircNCX1 promotes cardiomyocyte apoptosis via the miR-133a-3p/CDIP1 [59]. Circ-SATB2 can regulate vascular smooth muscle cell phenotypic differentiation, proliferation, apoptosis, and migration by promoting the expression of STIM1 (a target gene of miR-939) [60]. CircCHFR facilitates the proliferation and migration of vascular smooth muscle via the miR-370/FOXO1/cyclin D1 pathway [61]. CircSlc8al can function as an endogenous sponge for miR-133a in cardiomyocytes, and it can regulate the serum response factor, connective tissue growth factor, adrenoceptor beta 1 , and adenylate cyclase 6 through directly intervening the knockdown and overexpression in heart failure [62]. CircNfix can suppress Ybx1 ubiquitindependent degradation and increase miR-214 activity. It also can promote cardiac regenerative repair and functional recovery after MI [63]. Overexpression of circHIPK3 reverses miR-29b-3p-induced inhibition of cardiac fibrosis proliferation and migration, and then alters the expression levels of miR-29b-3p-targeting genes (a-SMA, COL1A1, COL3A1) in vitro [64]. 


\section{Potential circRNA-miRNA-mRNA Network in CHD Treated with TCM}

TCM may play a positive role in the treatment of cardiovascular diseases through the circRNA/miRNA/mRNA regulatory network (Figure 1). In myocardial ischemiareperfusion injury, increasing amounts of circNCX1 competitively bind to the miRNA-133a family, decreasing miRNA-133a-3p levels and increasing the activity of CDIP1, leading to cardiomyocyte deterioration [59]. Tongxinluo can increase the expression of the miRNA-133a family, weaken myocardial fibrosis, and reduce the damage caused by myocardial ischemia [65]. Therefore, Tongxinluo may play a regulatory role through the network of circNCX1/miRNA133a/mRNA. Modulation of circHRCR/miR-223/ARC levels provides an attractive therapeutic target for the treatment of cardiac hypertrophy and heart failure [57]. TCM that tonifies $Q i$ and activates blood (Shenmai injection, Shenqi Fuzheng injection, Danhong injection, and Qishen Yiqi pills) can significantly reduce the expression of miR223-3p in the plasma of patients with AMI. Studies have also found that activating blood and dissolving phlegm can significantly reduce the expression level of miR-223 in the serum of hyperlipidemia rats in the myocardial ischemiareperfusion model and play an anti-inflammatory role through the miR-223/PPRs pathway and inhibit apoptosis [66]. Ligustrazine, which is tetramethylpyrazine (TMP), can inhibit the proliferation, migration, apoptosis, and vascular remodeling of smooth muscle cells by downregulating the gene expression of miR-223 and then inhibiting the expression of CaMKII $\delta$ [67]. Ginsenoside Rg1 upregulates the expression of eNOS by targeting miR-214, which is beneficial for promoting angiogenesis [68]. Electroacupuncture preconditioning can upregulate the expression of miR-214 and inhibit the increase in $\mathrm{Ca}^{2+}$, sodium/calcium exchanger 1 (NCX1), BCL2-like 11 (BIM), calmodulin-dependent protein kinase II $\delta(\mathrm{CaMKII} \delta)$, cyclophilin D (CypD), and other related proteins that have accumulated in hypoxia injury in $\mathrm{H} 9 \mathrm{c} 2$ myocardial cells, and play a protective role in ische$\mathrm{mia} /$ reperfusion (I/R) [69]. The Fuzheng Huayu capsule has the effect of alleviating myocardial fibrosis after MI in rats. This mechanism may be related to upregulation of the expression levels in the miRNA-29 family and direct or indirect regulation of the ratio of matrix metalloproteinase $2 /$ tissue inhibitor of metalloproteinases 2 (MMP2/TIMP2) and MMP9/TIMP1, improving the metabolic balance in the extracellular matrix [70].

\section{Conclusion}

Currently, the main method used to treat CHD in China is to diagnose and treat the disease using modern medicine to determine syndrome differentiation and combine that with the usage of TCM [71]. Numerous studies have confirmed the role of circRNAs and miRNAs in the regulation of the formation and development of AS. CircRNA and miRNA play important roles not only in the regulation of the vascular structure and function but also in the process of CHD and AS. As previously mentioned, circRNA targets are circANRIL (PES1), CDR1as (PARP, SP1), circRNA-HRCR (ARC), MFACR (MTP18), circRNA_010567 (TGF- $\beta 1$ ), circNCX1 (CDIP1), circ-SATB2 (STIM1), circCHFR (FOXO1/CyclinD1), circNfix (Gsk3 $\beta$ ), and circHIPK3 (aSMA, COL1A1, COL3A1). Substantial research has been performed showing that miRNAs coordinate their mutual interaction between chronic endothelial repair and endothelial senescence and mechanistically link the regulation of macrophage cholesterol homeostasis with defective efferocytosis. MiRNA and its target gene (s) are let-7a-5p and let7b-5p (LOX-1), let-7g-5p (LOX-1, TGFBR1 and SMAD2), miR-26a-5p (TRPC6), miR-126-3p (RGS16), miR-126-5p (DLK1), miR-217-5p (SIRT1), miR-216a-5p (BECN1), miR34a-5p (SIRT1), miR-181b-5p (IPOA3), miR-92a-3p (KLF2, KLF4, SOCS5, and SIRT1), and miR-712-5p (Timp3) in the development of AS.

Single herb medicine, effective components, formulae, and Chinese patent medicines are widely used in the prevention and treatment of CHD. The functions of TCM in clinical treatment mainly include tonifying and regulating Qi, activating blood circulation, reducing phlegm, and relieving pain and consumptive disease. The treatment principles of PBSS in CHD are activating blood circulation and reducing phlegm, which are potential regulators of lipid metabolism and antiplatelet aggregation. However, due to the complex chemical components in CHM, its efficacy is actually a combination of various factors. Therefore, the biggest challenge for the modernization of TCM is to reduce the ambiguity of effective substances and mechanisms.

The circRNA-miRNA-mRNA network may be a new regulatory mechanism. CircRNAs and miRNAs interact with each other to regulate the mRNA expression of some critical genes in CHD genesis and progression. Previous studies showed that TCM contains active components that protect cardiomyocytes, act against atherosclerosis, inhibit apoptosis, promote cell growth, and protect the cardiovascular system by regulating miRNAs. Because there is a very tight connection among circRNAs, miRNAs, and mRNAs, it is likely that TCM may play a positive role in the treatment of CHD through the circRNA-miRNA-mRNA network. In conclusion, the circRNA-miRNA-mRNA network may be an important mechanism that can be affected by Chinese medicine, resulting in the amelioration of CHD. TCM can also be used to affect the circRNA-miRNA-mRNA network to assist with the diagnosis of CHD and the evaluation of the clinical effect of Chinese medicine on CHD.

\section{Conflicts of Interest}

The authors declare that they have no conflicts of interest.

\section{Authors' Contributions}

Fei Lin and Heng-Wen Chen contributed equally to this work. 


\section{References}

[1] S. S. Hu, R. L. Gao, L. S. Liu et al., "Summary of 2018 report on cardiovascular diseases in China," Chinese Circulation Journal, vol. 34, no. 3, pp. 6-17, 2019, in Chinese.

[2] C. P. Nelson, S. E. Hamby, D. Saleheen et al., "Genetically determined height and coronary artery disease," New England Journal of Medicine, vol. 372, no. 17, pp. 1608-1618, 2015.

[3] K. S. Manning and T. A. Cooper, "The roles of RNA processing in translating genotype to phenotype," Nature Reviews Molecular Cell Biology, vol. 18, no. 2, pp. 102-114, 2017.

[4] Z.-D. Wang, L.-Q. Jia, J. Wu et al., "Based on gene-constitutionsyndrome research model to discuss syndromes of coronary heart disease," Chinese Archives of Traditional Chinese Medicine, vol. 34, no. 5, pp. 1114-1117, 2016.

[5] Z.-Y. Zhou, Internal Medicine of Traditional Chinese Medicine, Chinese Medicine Press, Beijing, China, 2007.

[6] Z.-Y. Zhou, Chinese Internal Medicine, Beijing China Press of Traditional Chinese Medicine, Beijing, China, 2009.

[7] C. Liu and Y. Huang, "Chinese herbal medicine on cardiovascular diseases and the mechanisms of action," Frontiers in Pharmacology, vol. 7, 469 pages, 2016.

[8] G. Wang, G. Dai, J. Song et al., "Lactone component from Ligusticum chuanxiong alleviates myocardial ischemia injury through inhibiting autophagy," Frontiers in Pharmacology, vol. 9, no. 301, 2018.

[9] L. S. Einbond, F. Manservisi, H.-A. Wu et al., "A transcriptomic analysis of turmeric: curcumin represses the expression of cholesterol biosynthetic genes and synergizes with simvastatin," Pharmacological Research, vol. 132, pp. 176-187, 2018.

[10] H. Tao, X. Wu, J. Cao et al., "Rhodiola species: a comprehensive review of traditional use, phytochemistry, pharmacology, toxicity, and clinical study," Medicinal Research Reviews, vol. 39, no. 5, pp. 1779-1850, 2019.

[11] G.-Z. Yi, Y.-Q. Qiu, Y. Xiao, and L.-x. Yuan, “The usefulness of xuefu zhuyu tang for patients with angina pectoris: a metaanalysis and systematic review," Evidence-Based Complementary and Alternative Medicine, vol. 2014, Article ID 521602, 11 pages, 2014.

[12] M. Li, C. Li, S. Chen et al., "Potential effectiveness of Chinese patent medicine Tongxinluo capsule for secondary prevention after acute myocardial infarction: a systematic review and meta-analysis of randomized controlled trials," Frontiers in Pharmacology, vol. 9, p. 830, 2018.

[13] R. Chen, Y. Xiao, M. Chen et al., "A traditional Chinese medicine therapy for coronary heart disease after percutaneous coronary intervention: a meta-analysis of randomized, double-blind, placebo-controlled trials," Bioscience Reports, vol. 38, no. 5, 2018.

[14] X.-J. Han and S.-G. Shen, "Study the nature of angina pectoris of coronary heart disease marked by phlegm and stagnated blood," Chinese Journal of Basic Medicine in Traditional Chinese Medicine, vol. 8, no. 10, pp. 53-54, 2002.

[15] Y.-F. Bi, X.-L. Wang, Z.-Q. Zhao et al., "Clinical epidemiological investigation on current syndrome characteristics of coronary heart disease in Chinese medicine," Journal of Traditional Chinese Medicine, vol. 58, no. 23, pp. 2013-2019, 2017.

[16] Y.-J. Liu, J.-Q. Hu, and L.-J. Jiang, "Research progress on the molecular mechanism of the syndrome of phlegm and blood stasis in coronary heart disease," Modernization of Traditional Chinese Medicine and Materia Medica-World Science and Technology, vol. 18, no. 5, pp. 791-799, 2016.
[17] Y. Li, C. Huo, T. Pan et al., "Systematic review regulatory principles of non-coding RNAs in cardiovascular diseases," Briefings in Bioinformatics, vol. 20, no. 1, pp. 66-76, 2019.

[18] G. Yu, The Research of microRNA Biomarkers of Unstable Angina Patients with Blood Stasis Syndrome, China Academy of Chinese Medical Sciences, Beijing, China, 2013.

[19] F. Lin, H.-W. Chen, Z.-L. Shi, G.-A. Zhao, and Y.-X. Sun, "Explore molecular mechanism of Chinese herbs with promoting blood circulation and resolving phlegm effects on myocardial ischemia reperfusion injury based on correlation between microRNA and mtDNA," Zhongguo Zhong yao za zhi, vol. 42, no. 5, pp. 1005-1010, 2017.

[20] J. Wang, K.-W. Yao, Y.-M. Liu et al., "Transcriptomic studies on coronary heart disease with blood stasis syndrome-research approaches and methods in terms of biomarker and disease symptoms," Chinese Journal of Experimental Traditional Medical Formulae, vol. 23, no. 19, pp. 1-5, 2017.

[21] S. Chuppa, M. Liang, P. Liu et al., "MicroRNA-21 regulates peroxisome proliferator-activated receptor alpha, a molecular mechanism of cardiac pathology in cardiorenal syndrome type 4," Kidney International, vol. 93, no. 2, pp. 375-389, 2017.

[22] H.-Z. Zhu, MicroRNA Different Expression on Coronary Heart Disease with Qi- Deficiency Blood Stasis Syndrome and QiDeficiency Phlegm Stasis Syndrome and Treatment of Tongguan Capsule, Guangzhou University of Chinese Medicine, Guangzhou, China, 2017.

[23] M. Li, The microRNA Study of Huoxuehuatan'anshen Prescription for Patients with Angina Pectoris in CHD Phlegm and Blood Stasis Syndrome, China Academy of Chinese Medical Sciences, Beijing, China, 2016.

[24] J. Yuan, H. Chen, D. Ge et al., "Mir-21 promotes cardiac fibrosis after myocardial infarction via targeting smad7," Cellular Physiology and Biochemistry, vol. 42, no. 6, pp. 2207-2219, 2017.

[25] B. Zhou and J.-W. Yu, "A novel identified circular RNA, circRNA_010567, promotes myocardial fibrosis via suppressing miR-141 by targeting TGF- $\beta 1$," Biochemical and Biophysical Research Communications, vol. 487, no. 4, pp. 769-775, 2017.

[26] S. Liu, Y. Yang, S. Jiang et al., "MiR-378a-5p regulates proliferation and migration in vascular smooth muscle cell by targeting CDK1," Frontiers in Genetics, vol. 10, 22 pages, 2019.

[27] Y. Song, M. Hou, Z. Li et al., "TLR4/NF- $\kappa$ B/Ceramide signaling contributes to Ox-LDL-induced calcification of human vascular smooth muscle cells," European Journal of Pharmacology, vol. 794, pp. 45-51, 2017.

[28] N. Tang, S. Jiang, Y. Yang et al., "Noncoding RNAs as therapeutic targets in atherosclerosis with diabetes mellitus," Cardiovascular Therapeutics, vol. 36, no. 4, Article ID e12436, 2018.

[29] S. Liu, Y. Yang, S. Jiang et al., "Understanding the role of noncoding RNA (ncRNA) in stent restenosis," Atherosclerosis, vol. 272, no. 272, pp. 153-161, 2018.

[30] L. Xv, L.-Q. Jia, Z. Zhang et al., "Exploration on scientific connotation of coronary heart disease (CHD) phlegm-turbidity and blood stasis syndrome and treating CHD from spleen based on microRNAs," Chinese Archives of Traditional Chinese Medicine, vol. 35, no. 9, pp. 2249-2252, 2017.

[31] Z.-M. Yang, Y.-Y. Chen, X.-R. Yang et al., "Bioinformatics analysis of differential expression profiles from serum microRNA in dyslipidemia patients with pi-deficiency syndrome," Chinese Journal of Integrated Traditional Chinese and Western Medicine, vol. 38, no. 3, pp. 310-315, 2018. 
[32] M. Jia, Y. Miao, F.-H. Zhao et al., "Significance of circulating microRNAs in the severity discrimination and prognosis evaluation of acute coronary syndrome patients with blood stasis syndrome," Chinese Journal of Integrated Traditional and Western Medicine, vol. 38, no. 3, pp. 300-305, 2018.

[33] W. Chen, X. Li, S. Guo et al., "Tanshinone IIA harmonizes the crosstalk of autophagy and polarization in macrophages via miR-375/KLF4 pathway to attenuate atherosclerosis," International Immunopharmacology, vol. 70, pp. 486-497, 2019.

[34] L. Zhang, Y. Wu, Y. Li et al., "Tanshinone IIA improves miR133 expression through MAPK ERK1/2 pathway in hypoxic cardiac myocytes," Cellular Physiology and Biochemistry, vol. 30, no. 4, pp. 843-852, 2012.

[35] Z. Zhang, Y. Li, C. Sheng, C. Yang, L. Chen, and J. Sun, "Tanshinone IIA inhibits apoptosis in the myocardium by inducing microRNA-152-3p expression and thereby downregulating PTEN," American Journal of Translational Research, vol. 8, no. 7, pp. 3124-3132, 2016.

[36] J. Lin and X. Zheng, "Salvianolate blocks apoptosis during myocardial infarction by down regulating miR-122-5p," Current Neurovascular Research, vol. 14, no. 4, pp. 323-329, 2018.

[37] L. Gong, H. Chang, J. Zhang, G. Guo, J. Shi, and H. Xu, "Astragaloside IV protects rat cardiomyocytes from hypoxiainduced injury by down-regulation of miR-23a and miR-92a," Cellular Physiology and Biochemistry, vol. 49, no. 6, pp. 2240-2253, 2018.

[38] Z. Liu, H. Wang, G. Hou, H. Cao, Y. Zhao, and B. Yang, "Notoginsenoside R1 protects oxygen and glucose deprivation-induced injury by upregulation of miR-21 in cardiomyocytes," Journal of Cellular Biochemistry, vol. 120, no. 6, pp. 9181-9192, 2019.

[39] X. Yan, J. Liu, H. Wu et al., "Impact of miR-208 and its target gene nemo-Like kinase on the protective effect of ginsenoside Rb1 in hypoxia/ischemia injuried cardiomyocytes," Cellular Physiology and Biochemistry, vol. 39, no. 3, pp. 1187-1195, 2016.

[40] J. Chang, X. Xue, C. Song, B. Liu, and L. Gao, "Ginkgolide B promotes cell growth in endothelial progenitor cells through miR-126 and the Akt signaling pathway," Molecular Medicine Reports, vol. 16, no. 4, pp. 5627-5632, 2017.

[41] X. Wang, Y. Liang, J. Shi, H. J. Zhu, and B. E. Bleske, "Crataegus special extract WS 1442 effects on eNOS and microRNA 155," Planta Medica, vol. 84, no. 15, pp. 10941100, 2018.

[42] Y. Fan, L. Liang, T. Zhang et al., "Effect of Compound Danshen Dropping Pills on heart of diabetic cardiomyopathy rats by regulating VEGF/TGF- $\beta 1$ by miRNA-200b," Chinese Journal of Experimental Traditional Medical Formulae, vol. 25, no. 17, pp. 34-42, 2019.

[43] Y. Qi, K. Zhu, X. Ren et al., "Institute of Basic Research in Clinical Medicine. Effects of Fuzheng Huayu Capsule on the expression of mir-29 family in rats with myocardial fibrosis after myocardial infarction," Global Traditional Chinese Medicine, vol. 12, no. 6, pp. 839-844, 2019.

[44] P. Guo, Y. Liang, Z. Chen, Y. Li, L. Sun, and K. Gao, "Effect of Xinfukang Pill on myocardial miRNA-21 of pressure overload rats," Chinese Journal of Experimental Traditional Medical Formulae, vol. 22, no. 21, pp. 137-141, 2016.

[45] Y. Liang, P. Guo, Z. Chen et al., "Experimental study of Xinfukang pills on microRNA-1 in cardiac tissue of pressure overloaded rats," Tianjin Journal of Traditional Chinese Medicine, vol. 34, no. 1, pp. 48-53, 2017.
[46] H. Zhang, S. Li, Q. Zhou et al., "Qiliqiangxin attenuates phenylephrine-induced cardiac hypertrophy through downregulation of MiR-199a-5p," Cellular Physiology and Biochemistry, vol. 38, no. 5, pp. 1743-1745, 2016.

[47] S. J. Conn, K. A. Pillman, J. Toubia et al., "The RNA binding protein quaking regulates formation of circRNAs," Cell, vol. 160, no. 6, pp. 1125-1134, 2015.

[48] X. M. Fan, X. Y. Weng, Y. F. Zhao, W. Chen, T. Gan, and D. Xu, "Circular RNAs in cardiovascular disease: an overview," Biomed Research International, vol. 2017, no. 6, Article ID 5135781, 9 pages, 2017.

[49] I. Legnini, G. Di Timoteo, F. Rossi et al., "Circ-ZNF609 is a circular RNA that can be translated and functions in myogenesis," Molecular Cell, vol. 66, no. 1, pp. 22-37, 2017.

[50] Y. Y. Wang and G. P. Li, "The emerging landscape of circular RNAs in cardiovascular diseases," Tianjin Medical Journal, vol. 45, no. 3, pp. 333-336, 2017.

[51] G.-A. Zhao, "Significance of non-coding circular RNAs and microRNAs in the pathogenesis of cardiovascular diseases," Journal of Medical Genetics, vol. 55, no. 11, pp. 713-720, 2018.

[52] J.-L. Gao, Study on Diagnostic Scale and Differential Expression of circRNA in Qi Stagnation and Blood Stasis Syndrome, China Academy of Chinese Medical Sciences, Beijing, China, 2017.

[53] F. Lin, G.-A. Zhao, Z.-G. Chen et al., "Network correlation of circRNA-miRNA and the possible regulatory mechanism in acute myocardial infarction," Zhonghua Yi Xue Za Zhi, vol. 98, no. 11, pp. 851-854, 2018.

[54] R.-Y. Pan, P. Liu, H.-T. Zhou et al., "Circular RNAs promote TRPM3 expression by inhibiting hsa-miR-130a-3p in coronary artery disease patients," Oncotarget, vol. 8, no. 36, pp. 60280-60290, 2017.

[55] L. M. Holdt, A. Stahringer, K. Sass et al., "Circular non-coding RNA ANRIL modulates ribosomal RNA maturation and atherosclerosis in humans," Nature Communications, vol. 7, p. 12429, 2016.

[56] H. H. Geng, L. Rui, Y. M. Su et al., "The Circular RNA cdr1as promotes myocardial infarction by mediating the regulation of miR-7a on its target genes expression," PLoS One, vol. 11, no. 3, Article ID e0151753, 2016.

[57] K. Wang, B. Long, F. Liu et al., "A circular RNA protects the heart from pathological hypertrophy and heart failure by targeting miR-223," European Heart Journal, vol. 37, no. 33, pp. 2602-2611, 2016.

[58] K. Wang, T. Y. Gan, N. Li et al., "Circular RNA mediates cardiomyocyte death via miRNA-dependent upregulation of MTP18 expression," Cell Death \& Differentiation, vol. 24, no. 6, pp. 1111-1120, 2017.

[59] M. Li, W. Ding, M. A. Tariq et al., "A circular transcript of ncxl gene mediates ischemic myocardial injury by targeting miR-133a-3p," Theranostics, vol. 8, no. 21, pp. 5855-5869, 2018.

[60] Y. Y. Mao, J. Q. Wang, X. X. Guo, Y. Bi, and C. X. Wang, "Circ-SATB2 upregulates STIM1 expression and regulates vascular smooth muscle cell proliferation and differentiation through miR-939," Biochemical and Biophysical Research Communications, vol. 505, no. 1, pp. 119-125, 2018.

[61] L. Yang, F. Yang, H. Zhao, M. Wang, and Y. Zhang, "Circular RNA circCHFR facilitates the proliferation and migration of vascular smooth muscle via miR-370/FOXO1/cyclin D1 pathway," Molecular Therapy-Nucleic Acids, vol. 16, pp. 434-441, 2019.

[62] T. B. Lim, E. Aliwarga, T. D. A. Luu et al., "Targeting the highly abundant circular rna circslc8a1 in cardiomyocytes 
attenuates pressure overload induced hypertrophy," Cardiovascular Research, vol. 115, no. 14, pp. 1998-2007, 2019.

[63] S. Huang, X. Li, H. Zheng et al., "Loss of super-enhancerregulated circRNA Nfix induces cardiac regeneration after myocardial infarction in adult mice," Circulation, vol. 139, no. 25, pp. 2857-2876, 2019.

[64] H. Ni, W. Li, Y. Zhuge et al., "Inhibition of circHIPK3 prevents angiotensin II-induced cardiac fibrosis by sponging miR-29b-3p," International Journal of Cardiology, vol. 292, pp. 188-196, 2019.

[65] W.-M. Luo, The Role and Mechanism of Unblocking and Collaterals Therapy on Target Organ Damages in Essential Hypertension, Shandong University, Jinan, China, 2016.

[66] J. W. Zhu, G. H. Dai, J. Yao et al., "Effect of Yiqi Huoxue Recipe on the expression of plasma MiR-223-3p and miR$132-5 p$ in patients with acute myocardial infarction," Chinese Journal of Integrative Medicine on Cardio-/Cerebrovascuiar Disease, vol. 14, no. 09, pp. 939-943, 2016.

[67] M. Yang, Effects of Tetramethylpyrazine on Calmodulin-dependent Protein Kinase II Delta Isoform Promote Rats Vascular Remodeling, Shanxi Provincial Institute of TCM, Shanxi, China, 2017.

[68] L. S. Chan, P. Y. Yue, N. K. Mak, and N. S. Wong, "Role of microRNA-214 in ginsenoside-Rg1-induced angiogenesis," European Journal of Pharmaceutical Sciences, vol. 38, no. 4, pp. 370-377, 2009.

[69] P. Y. Liu, Y. Tian, and S. Y. Xu, "Mediated protective effect of electroacupuncture pretreatment by miRNA-214 on myocardial ischemia/reperfusion injury," Journal of Geriatric Cardiology, vol. 11, no. 4, pp. 303-310, 2014.

[70] W. W. Liu, Exosomal circHIPK3 Released from Cardiomyocytes Pretreated with Hypoxia Regulates Apoptosis and Proliferation of Cardiac Microvascular Endothelial Cells under Oxidative Stress, Zunyi Medical University, Zunyi, China, 2019.

[71] W. Jie, L. Fei, L. L. Guo, X. J. Xiong, and F. Xun, "Cardiovascular disease, mitochondria, and traditional chinese medicine," Evidence-Based Complementary and Alternative Medicine, vol. 2015, Article ID 143145, 7 pages, 2015. 